\title{
The QED vacuum polarization function at four loops and the anomalous magnetic moment at five loops
}

\author{
P. Baikov ${ }^{\mathrm{a}}$, A. Maier ${ }^{\mathrm{b}}$, P. Marquard ${ }^{\mathrm{c}}$ \\ ${ }^{a}$ Skobeltsyn Institute of Nuclear Physics, Lomonosov Moscow State University, 1(2), \\ Leninskie gory, Moscow 119991, Russian Federation \\ ${ }^{b}$ Physik Department T31, James-Franck-Straße, Technische Universität München, D85748 \\ Garching, Germany \\ ${ }^{c}$ Deutsches Elektronen Synchrotron DESY, Platanenallee 6, D15637 Zeuthen, Germany
}

\begin{abstract}
The anomalous moment of the muon is one of the most fundamental observables. It has been measured experimentally with a very high precision and on theory side the contributions from perturbative QED have been calculated up to five-loop level by numerical methods. Contributions to the muon anomalous magnetic moment from certain diagram classes are also accessible by alternative methods. In this paper we present the evaluation of contributions to the QCD corrections due to insertions of the vacuum polarization function at five-loop level.
\end{abstract}

Keywords: Perturbative calculations, QED, lepton anomalous moment

\section{Introduction}

The anomalous magnetic moments of electron and muon are among the best experimentally measured quantities and are also very well understood from theoretical side. In particular, the perturbative QED corrections have been under consideration for a very long time. The leading-order QED corrections have been considered by Schwinger in Ref. [1], the next-to-leading order corrections not much later in Refs. [2, 3]. At three loops, i.e. next-to-next-to-leading order, the QED corrections have been first calculated numerically in Ref. 4] and later analytically in Refs. [5 7]. At four and five loops the complete QED contributions have been calculated numerically in Refs. [8, 9] while only partial analytical results exist [10 18]. For a more thorough review of the current status see e.g. Ref. [19].

In the case of the muon anomalous moment, next to the universal contribution, contributions from diagrams with closed electron loops are of particular interest. A subgroup of this class of diagrams, contributions from corrections to the photon propagator, can be easily obtained using already available building blocks [10, 20]. At five loops the leading contributions have been obtained from considering the asymptotic form [17, which resulted in unexpected discrepancies with the results obtained in Ref.[21]. The aim of this paper is to improve the 
predictions for the anomalous magnetic moment of the muon made in Ref. [17] and to resolve the discrepancies. To this extend we approximately reconstruct the photon vacuum polarization at four loops using all available information.

This paper is organized as follows: In Section 2 we collect the necessary information to construct an approximation for the vacuum polarization function at four loops. In Section 3 we present the calculation of the contribution to the anomalous magnetic muon from vacuum polarization insertions.

\section{QED vacuum polarization at four loops}

We define the vacuum polarization function $\Pi\left(q^{2}\right)$ as usual by

$$
\left(q^{\mu} q^{\nu}-q^{2} g^{\mu \nu}\right) \Pi\left(q^{2}\right)=i \int \mathrm{d} x\left\langle 0\left|e^{i q x} T j^{\mu}(x) j^{\nu}(0)\right| 0\right\rangle
$$

with the current $j^{\mu}=\bar{\psi} \gamma^{\mu} \psi$ and write it as an expansion in the fine-structure constant $\alpha$

$\Pi\left(q^{2}\right)=\frac{\alpha}{\pi} \Pi^{(1)}\left(q^{2}\right)+\left(\frac{\alpha}{\pi}\right)^{2} \Pi^{(2)}\left(q^{2}\right)+\left(\frac{\alpha}{\pi}\right)^{3} \Pi^{(3)}\left(q^{2}\right)+\left(\frac{\alpha}{\pi}\right)^{4} \Pi^{(4)}\left(q^{2}\right)+\mathcal{O}\left(\alpha^{5}\right)$.

At three and four loops the vacuum polarization can only be calculated in a low-energy, high-energy or threshold expansion at the moment. The available information can then be used to construct an approximate function using Padé approximation. In the following we will present the results for the corresponding expansions and construct an approximating function. The renormalization of lepton mass and fine-structure constant $\alpha$ is performed in the on-shell scheme. Note that in this scheme the condition $\Pi(0)=0$ is being imposed.

\subsection{Low-energy expansion}

In the low-energy limit the polarization function can be expanded in a power series in $z=q^{2} /\left(4 m_{q}^{2}\right)<1$

$$
\Pi_{\mathrm{le}}^{(n)}=\frac{1}{16 \pi^{2}} \sum_{k=1} \Pi_{\mathrm{le}, k}^{(n)} z^{k} .
$$

For the QED case at hand, the result for the non-singlet contribution can in principle be obtained from the QCD results given in Ref. [22]. But since the results are not given expressed through colour factors they cannot easily be translated to the case of QED. The low-energy expansion was therefore recalculated for the case at hand. The calculation follows a well-established path. The Feynman diagrams are generated using qgraf [23] and mapped onto six topologies using q2e and exp [24, 25]. Then a FORM [26] program is used to apply projectors and take traces. The resulting scalar integrals are then reduced to master integrals using Crusher [27], which implements Laporta's algorithm [28] for solving integration-by-parts identities. The needed master integrals have 
been calculated in Refs. 29 37]. Combining all steps and performing the renormalization of all quantities in the on-shell scheme, the first three moments are obtained

$$
\begin{aligned}
& \Pi_{\mathrm{le}, 1}^{(4)}=\left(-\frac{30853 a_{1}^{4}}{22680}+\frac{30853 \pi^{2} a_{1}^{2}}{22680}-\frac{64 \pi^{2} a_{1}}{135}-\frac{30853 a_{4}}{945}-\frac{77255063 \zeta_{3}}{4762800}\right. \\
& \left.+\frac{473237 \pi^{4}}{1360800}+\frac{866 \pi^{2}}{1215}-\frac{3619201}{529200}\right) n_{l}^{2}+\left(\frac{3376 a_{1}^{5}}{2835}-\frac{2178299 a_{1}^{4}}{544320}-\frac{3376 \pi^{2} a_{1}^{3}}{1701}\right. \\
& +\frac{2178299 \pi^{2} a_{1}^{2}}{544320}-\frac{12191 \pi^{4} a_{1}}{8505}-\frac{1922 \pi^{2} a_{1}}{405}-\frac{2178299 a_{4}}{22680}-\frac{27008 a_{5}}{189}+\frac{\pi^{2} \zeta_{3}}{30} \\
& \left.-\frac{69694097 \zeta_{3}}{1209600}+\frac{33931 \zeta_{5}}{189}+\frac{66975707 \pi^{4}}{65318400}+\frac{7297 \pi^{2}}{3240}+\frac{3562169}{2721600}\right) n_{l}+ \\
& \mathbf{s i}\left(-\frac{739 a_{1}^{4}}{1080}+\frac{739 \pi^{2} a_{1}^{2}}{1080}-\frac{739 a_{4}}{45}-\frac{2017831 \zeta_{3}}{213840}+\frac{35 \pi^{4}}{216}+\frac{664837}{641520}\right) n_{l}^{2} \\
& +\left(-\frac{1291 \zeta_{3}}{1944}-\frac{32 \pi^{2}}{2025}+\frac{83971}{78732}\right) n_{l}^{3}, \\
& \Pi_{\mathrm{le}, 2}^{(4)}=\left(-\frac{130829911 a_{1}^{4}}{8709120}+\frac{130829911 \pi^{2} a_{1}^{2}}{8709120}-\frac{128 \pi^{2} a_{1}}{315}-\frac{130829911 a_{4}}{362880}\right. \\
& \left.-\frac{5763324918049 \zeta_{3}}{26824089600}+\frac{2208846791 \pi^{4}}{522547200}+\frac{10846 \pi^{2}}{14175}-\frac{42466787908001}{1086375628800}\right) n_{l}^{2} \\
& +\left(\frac{19593724 a_{1}^{5}}{2027025}-\frac{60530131639 a_{1}^{4}}{24908083200}-\frac{19593724 \pi^{2} a_{1}^{3}}{1216215}+\frac{60530131639 \pi^{2} a_{1}^{2}}{24908083200}\right. \\
& -\frac{72540947 \pi^{4} a_{1}}{6081075}-\frac{3058 \pi^{2} a_{1}}{675}-\frac{60530131639 a_{4}}{1037836800}-\frac{156749792 a_{5}}{135135}+\frac{\pi^{2} \zeta_{3}}{35} \\
& \left.-\frac{112244692092317 \zeta_{3}}{6974263296000}+\frac{14315837 \zeta_{5}}{10010}-\frac{427149037853 \pi^{4}}{2988969984000}+\frac{839 \pi^{2}}{378}+\frac{94330906317547}{3487131648000}\right) n_{l} \\
& +\mathbf{s i}\left(\frac{97011619 a_{1}^{4}}{174182400}-\frac{97011619 \pi^{2} a_{1}^{2}}{174182400}+\frac{97011619 a_{4}}{7257600}+\frac{796232393699 \zeta_{3}}{92990177280}-\frac{745372259 \pi^{4}}{4180377600}\right. \\
& \left.+\frac{5881974201847}{2092278988800}\right) n_{l}^{2}+\left(-\frac{19669747 \zeta_{3}}{21288960}-\frac{64 \pi^{2}}{4725}+\frac{3284183491}{2586608640}\right) n_{l}^{3}
\end{aligned}
$$




$$
\begin{aligned}
& \Pi_{\mathrm{le}, 3}^{(4)}=\left(-\frac{1875259367 a_{1}^{4}}{17740800}+\frac{1875259367 \pi^{2} a_{1}^{2}}{17740800}-\frac{1024 \pi^{2} a_{1}}{2835}-\frac{1875259367 a_{4}}{739200}\right. \\
& \left.-\frac{377287031234107 \zeta_{3}}{245887488000}+\frac{95566793477 \pi^{4}}{3193344000}+\frac{1175348 \pi^{2}}{1488375}-\frac{2388270016962373}{9958443264000}\right) n_{l}^{2} \\
& +\left(\frac{12135758968 a_{1}^{5}}{172297125}-\frac{245658223193 a_{1}^{4}}{6616209600}-\frac{12135758968 \pi^{2} a_{1}^{3}}{103378275}+\frac{245658223193 \pi^{2} a_{1}^{2}}{6616209600}\right. \\
& -\frac{45718624634 \pi^{4} a_{1}}{516891375}-\frac{720316 \pi^{2} a_{1}}{165375}-\frac{245658223193 a_{4}}{275675400}-\frac{97086071744 a_{5}}{11486475}+\frac{8 \pi^{2} \zeta_{3}}{315} \\
& -\frac{1531450738927589 \zeta_{3}}{3368252160000}+\frac{119140260224 \zeta_{5}}{11486475}+\frac{176076905389817 \pi^{4}}{31757806080000}+\frac{5337 \pi^{2}}{2450} \\
& \left.+\frac{300002162759308069}{1500556337280000}\right) n_{l}+\mathbf{s i}\left(\frac{22845879073 a_{1}^{4}}{6096384000}-\frac{22845879073 \pi^{2} a_{1}^{2}}{6096384000}\right. \\
& \left.+\frac{22845879073 a_{4}}{254016000}+\frac{343009147408727 \zeta_{3}}{6246309888000}-\frac{787819133821 \pi^{4}}{731566080000}+\frac{7118016595194017}{758926651392000}\right) n_{l}^{2} \\
& +\left(-\frac{7731286469 \zeta_{3}}{6227020800}-\frac{512 \pi^{2}}{42525}+\frac{81866930683}{50438868480}\right) n_{l}^{3},
\end{aligned}
$$

where $n_{l}$ denotes contributions from closed lepton loops and where we used the abbreviations $a_{1}=\log 2$ and $a_{n}=\operatorname{Li}_{n}(1 / 2)$. si marks contributions from singlet diagrams.

\subsection{High-energy expansion}

In the high-energy region we write the result in the form

$$
\Pi_{\mathrm{he}}^{(n)}=\frac{1}{16 \pi^{2}} \sum_{k=0} \Pi_{\mathrm{he}, k}^{(n)} z^{-k} .
$$

The coefficients of Eq. (7) can be expressed through four-loop massless propagator integrals which, in turn, can be reduced to 28 master integrals. This reduction has been done by evaluating sufficiently many terms of the $1 / D$ expansion [38] of the corresponding coefficient functions [39]. The master integrals are known analytically from [40]. 
As the result, the leading two coefficients of Eq. (7) are

$$
\begin{aligned}
& \Pi_{\mathrm{he}, 0}^{(4)}=\mathbf{s i}\left(\frac{73 a_{1}^{4}}{144}-\frac{73}{144} \pi^{2} a_{1}^{2}+\frac{73 a_{4}}{6}-\frac{2 \zeta_{3}^{2}}{3}+\frac{5309 \zeta_{3}}{1120}+\frac{5 \zeta_{5}}{3}+\frac{2}{3} \zeta_{3} \log (-4 z)\right. \\
& \left.-\frac{11}{36} \log (-4 z)-\frac{2237 \pi^{4}}{17280}+\frac{1963}{3780}\right) n_{l}^{2}+n_{l}^{2}\left(\frac{53 a_{1}^{4}}{60}-\frac{53}{60} \pi^{2} a_{1}^{2}+\frac{16 \pi^{2} a_{1}}{27}+\frac{106 a_{4}}{5}-\zeta_{3}^{2}\right. \\
& +\frac{29129 \zeta_{3}}{1800}-\frac{125 \zeta_{5}}{18}-\frac{19}{12} \zeta_{3} \log (-4 z)+\frac{5}{3} \zeta_{5} \log (-4 z)-\frac{1}{48} \log ^{2}(-4 z)-\frac{1}{12} \log (-4 z) \\
& \left.-\frac{2161 \pi^{4}}{10800}-\frac{179 \pi^{2}}{324}+\frac{3361}{900}\right)+n_{l}\left(-\frac{32 a_{1}^{5}}{225}+\frac{1559 a_{1}^{4}}{1080}+\frac{32}{135} \pi^{2} a_{1}^{3}-\frac{1559 \pi^{2} a_{1}^{2}}{1080}\right. \\
& +\frac{106 \pi^{4} a_{1}}{675}+\frac{59 \pi^{2} a_{1}}{12}+\frac{1559 a_{4}}{45}+\frac{256 a_{5}}{15}-\frac{\pi^{2} \zeta_{3}}{24}+\frac{6559 \zeta_{3}}{320}-\frac{1603 \zeta_{5}}{120}-\frac{35 \zeta_{7}}{4} \\
& \left.+\frac{23}{128} \log (-4 z)-\frac{59801 \pi^{4}}{129600}-\frac{157 \pi^{2}}{72}-\frac{71189}{34560}\right)+n_{l}^{3}\left(-\frac{15109 \zeta_{3}}{22680}-\frac{5 \zeta_{5}}{9}\right. \\
& -\frac{1}{9} \zeta_{3} \log ^{2}(-4 z)+\frac{19}{27} \zeta_{3} \log (-4 z)-\frac{1}{108} \log ^{3}(-4 z)+\frac{11}{72} \log ^{2}(-4 z)-\frac{151}{162} \log (-4 z) \\
& \left.+\frac{8 \pi^{2}}{405}+\frac{75259}{68040}\right) \\
& \Pi_{\mathrm{he}, 1}^{(4)}=n_{l}^{2}\left(\frac{a_{1}^{4}}{9}-\frac{1}{9} \pi^{2} a_{1}^{2}+\frac{8 \pi^{2} a_{1}}{9}+\frac{8 a_{4}}{3}-\frac{\zeta_{3}^{2}}{6}+\frac{3023 \zeta_{3}}{432}-\frac{415 \zeta_{5}}{54}\right. \\
& -\frac{167}{72} \zeta_{3} \log (-4 z)-\frac{35}{36} \zeta_{5} \log (-4 z)+\frac{3}{16} \log ^{3}(-4 z)-\frac{15}{16} \log ^{2}(-4 z)+\frac{1}{4} \pi^{2} \log (-4 z) \\
& \left.+\frac{539}{144} \log (-4 z)+\frac{5 \pi^{4}}{108}-\frac{103 \pi^{2}}{108}-\frac{491}{216}\right)+n_{l}\left(-\frac{3}{4} \pi^{2} a_{1} \log (-4 z)-\frac{a_{1}^{4}}{2}+\frac{1}{2} \pi^{2} a_{1}^{2}\right. \\
& +\frac{31 \pi^{2} a_{1}}{4}-12 a_{4}-2 \zeta_{3}^{2}-\frac{\pi^{2} \zeta_{3}}{16}-\frac{3647 \zeta_{3}}{192}-\frac{1825 \zeta_{5}}{192}+\frac{10073 \zeta_{7}}{384}+\frac{41}{32} \zeta_{3} \log (-4 z) \\
& +\frac{35}{16} \zeta_{5} \log (-4 z)-\frac{9}{32} \log ^{3}(-4 z)+\frac{9}{64} \log ^{2}(-4 z)+\frac{15}{32} \pi^{2} \log (-4 z)-\frac{685}{128} \log (-4 z) \\
& \left.-\frac{\pi^{4}}{48}-\frac{673 \pi^{2}}{192}-\frac{47}{36}\right)+\mathbf{s i}\left(-\frac{9 \zeta_{3}^{2}}{2}-\frac{13 \zeta_{3}}{3}-\frac{5 \zeta_{5}}{12}+\frac{147 \zeta_{7}}{16}+\frac{1}{3}\right) n_{l}^{2} \\
& +n_{l}^{3}\left(-\frac{4 \zeta_{3}}{9}+\frac{2}{3} \zeta_{3} \log (-4 z)-\frac{1}{36} \log ^{3}(-4 z)+\frac{13}{72} \log ^{2}(-4 z)-\frac{317}{216} \log (-4 z)\right. \\
& \left.+\frac{4 \pi^{2}}{135}+\frac{25}{27}\right)
\end{aligned}
$$

where $n_{l}$ denotes contributions from closed lepton loops and where we used the abbreviations $a_{1}=\log 2$ and $a_{n}=\operatorname{Li}_{n}(1 / 2)$. si marks contributions from singlet diagrams. 


\subsection{Threshold expansion}

The polarization function in the threshold region can be written as

$$
\Pi_{\mathrm{thr}}^{(n)}=\sum_{k=2-n} \Pi_{\mathrm{thr}, k}^{(n)} \sqrt{1-z}^{k} .
$$

In non-relativistic quantum field theory the NNLO expression for the threshold cross section is known for an arbitrary $S U(N)$ gauge group [41]. This means that the derivation of the threshold expansion is essentially the same as for the QCD case, which is described in Refs. [42, 43]. The only additional steps consist of setting the group invariants to their $U(1)$ values and converting the coupling to the on-shell scheme.

The resulting coefficients read

$$
\begin{aligned}
\Pi_{\mathrm{thr},-1}^{(3)}= & \frac{\pi^{3}}{384} n_{l}, \\
\Pi_{\mathrm{thr}, 0}^{(3)}= & \left(\frac{17}{768}+\frac{11}{128} a_{1}\right) n_{l} \log (1-z)+\frac{11}{512} n_{l} \log ^{2}(1-z)+\mathrm{const}, \\
\Pi_{\mathrm{thr}, 1}^{(3)}= & \left(\frac{17}{192 \pi}-\frac{397}{2304} \pi+\frac{5}{24} \pi a_{1}-\frac{13}{2304} \pi^{3}+\frac{41}{64 \pi} \zeta_{3}\right) n_{l} \\
& +\frac{11}{192} \pi n_{l} \log (1-z)+\left(-\frac{11}{72 \pi}+\frac{\pi}{72}\right) n_{l}^{2},
\end{aligned}
$$

for $n=3$ and

$$
\begin{aligned}
\Pi_{\mathrm{thr},-2}^{(4)}= & \frac{1}{128} \pi^{2} \zeta_{3} n_{l} \\
\Pi_{\mathrm{thr},-1}^{(4)}= & \left(\frac{7}{2304} \pi^{3}-\frac{11}{384} \pi^{3} a_{1}-\frac{11}{64} \pi \zeta_{3}\right) n_{l}-\frac{11}{768} \pi^{3} n_{l} \log (1-z) \\
\Pi_{\mathrm{thr}, 0}^{(4)}= & \left(-\frac{467}{18432}+\frac{115}{384} a_{1}-\frac{121}{256} a_{1}^{2}-\frac{2207}{9216} \pi^{2}+\frac{5}{48} \pi^{2} a_{1}\right. \\
& \left.+\frac{9}{4096} \pi^{4}+\frac{131 \zeta_{3}}{512}\right) n_{l} \log (1-z) \\
& +\left(-\frac{839}{9216}+\frac{\pi^{2}}{144}\right) n_{l}^{2} \log (1-z) \\
& +\left(\frac{115}{1536}-\frac{121}{512} a_{1}+\frac{11}{768} \pi^{2}\right) n_{l} \log ^{2}(1-z) \\
& -\frac{121}{3072} n_{l} \log ^{3}(1-z)+\mathrm{const}
\end{aligned}
$$

for $n=4$, where we again used $a_{1}=\log 2$.

\subsection{Padé approximation}

Having all building blocks at hand the polarization function can be reconstructed using Padé approximation [15, 42 48]. For the four-loop contribution 
we closely follow the procedure outlined in Ref. [43]. In the three-loop case we introduce slight modifications to accommodate the large amount of information in the available low- and high-energy expansions. We first give a brief review of the method as used in the four-loop case and then discuss the changes for the application to the three-loop contribution.

\subsubsection{Approximation procedure at four loops}

As in Ref. [43], we first split $\Pi^{(4)}(z)$ into two parts,

$$
\Pi^{(4)}(z)=\Pi_{\text {reg }}^{(4)}(z)+\Pi_{\log }^{(4)}(z),
$$

using the ansatz

$$
\begin{aligned}
& \Pi_{\log }^{(4)}(z)=\sum_{j=-1}^{0} \sum_{i=1}^{3+2 j} k_{i j} \mathcal{K}_{i j}^{(4)}(z)+\sum_{n=0}^{1} \sum_{m=0}^{3} d_{m n} \mathcal{D}_{m n}^{(4)}(z), \\
& \mathcal{K}_{i j}^{(4)}(z)=\Pi^{(2)}(z)^{i} G(z)^{j} \times\left\{\begin{array}{cc}
\left(A_{1}+\frac{1}{z}\right) & \text { for } i=3, j=0 \\
\left(A_{2}+\frac{1}{z}\right) & \text { for } i=1, j=-1, \\
1 & \text { otherwise }
\end{array}\right. \\
& \mathcal{D}_{m n}^{(4)}(z)=(z G(z))^{m}(1-1 / z)^{\left\lceil\frac{m}{2}\right\rceil} \frac{1}{z^{n}} \times\left\{\begin{array}{cc}
\left(1+\frac{1}{B_{1} z}\right) & \text { for } n=3 \\
\left.1+\frac{1}{B_{2} z}\right) & \text { for } n=2 \\
1 & \text { otherwise }
\end{array}\right.
\end{aligned}
$$

with the known two-loop polarization function $\Pi^{(2)}(z)$ as introduced in Eq. (2) and

$$
G(z)=\frac{1}{2 z} \frac{\log (u)}{\sqrt{1-\frac{1}{z}}}, \quad u=\frac{\sqrt{1-\frac{1}{z}}-1}{\sqrt{1-\frac{1}{z}}+1} .
$$

The coefficients $k_{i j}$ can be fixed so that all known logarithms in the threshold expansion (Eqs. (10), (14)- (16)) are absorbed into the first term on the righthand side of Eq. (18). Expanding Eq. (19) in the threshold region generates the

required logarithms and half-integer powers of $1-z$, as can be seen from the expansions

$$
\begin{gathered}
G(z)=\frac{\pi}{2} \frac{1}{\sqrt{1-z}}+\mathcal{O}\left((1-z)^{0}\right), \\
\Pi^{(2)}(z)=-\frac{1}{16} \log (1-z)+\text { const }+\mathcal{O}(\sqrt{1-z}) .
\end{gathered}
$$

In a similar way the factor $z G(z)$ in Eq. (20) generates logarithms in the high-energy region:

$$
z G(z)=-\frac{1}{2} \log (-4 z)+\mathcal{O}\left(\frac{1}{z}\right)
$$


This means we can choose the coefficients $d_{m n}$ with $m>0$ in such a way that these logarithms are also absorbed into $\Pi_{\log }^{(4)}(z)$. The remaining coefficients $d_{0 n}$ are fixed by requiring that $\Pi_{\text {reg }}^{(4)}(z)$ has no poles for $z \rightarrow 0$ and, more specifically, $\Pi_{\text {reg }}^{(4)}(0)=0$.

To estimate the error of the approximation we vary the parameters $A_{i}, B_{i}$ independently with

$$
\begin{aligned}
& A_{i} \in\{-1 \pm 1,-1 \pm 4,-1 \pm 16,-1 \pm 64\} \\
& B_{i} \in\{ \pm 1, \pm 4, \pm 16, \pm 64\} .
\end{aligned}
$$

In a second step we define

$$
P_{1}(\omega)=\left(\frac{1-\omega}{1+\omega}\right)^{2}\left(\Pi_{\mathrm{reg}}^{(4)}(z(w))-\Pi_{\mathrm{reg}}^{(4)}(-\infty)\right)
$$

with $z(\omega)=4 \omega /(1+\omega)^{2}$ and construct Padé approximants

$$
p_{n, m}(\omega)=\frac{\sum_{i=0}^{n-1} a_{i} \omega^{i}+\omega^{n}}{\sum_{i=0}^{m} b_{i} \omega^{i}}
$$

using the constraints

$$
\begin{aligned}
& p_{n, m}(1)=P_{1}(1), \quad p_{n, m}^{\prime}(1)=P_{1}^{\prime}(1), \\
& p_{n, m}(-1)=P_{1}(-1), \quad p_{n, m}^{(i)}(0)=P_{1}^{(i)}(0), \quad i=0, \ldots, 3,
\end{aligned}
$$

and requiring the absence of terms proportional to $z^{-1 / 2}$ in the high-energy expansion of $\Pi_{\text {reg. }}^{(4)}$. In total there are eight constraints, i.e. we obtain Padé approximants with $n+m=7$.

If $p_{n, m}(\omega)$ has poles inside the unit circle $|\omega|<1$ the corresponding reconstructed polarization function shows unphysical singularities in the complex $z$ plane. We therefore discard such approximants. Furthermore, we require $\left|p_{n, m}(\omega)\right|<1.5$ for $|\omega|=1$ in order to remove approximants with pronounced additional peaks above the physical threshold at $z=1$. A notable effect of this cut is the elimination of all Taylor approximants $p_{7,0}(\omega)$.

Since the approximants are only available in numerical form we show the general features in Fig. 1. At the top of the figure we show the envelope of all Padé approximants that have been calculated and compare with the lowand high-energy expansions. In the bottom half we show the maximal deviation from the mean in percent. As can be seen there are only significant deviations in the range $x>0.8$, which correspond to the uncertainty due to a limited number of terms in the high-energy expansion.

\subsubsection{Modifications at three loops}

At three loops much deeper expansions can be used for the construction of the Padé approximants and solving the resulting large system of constraints 

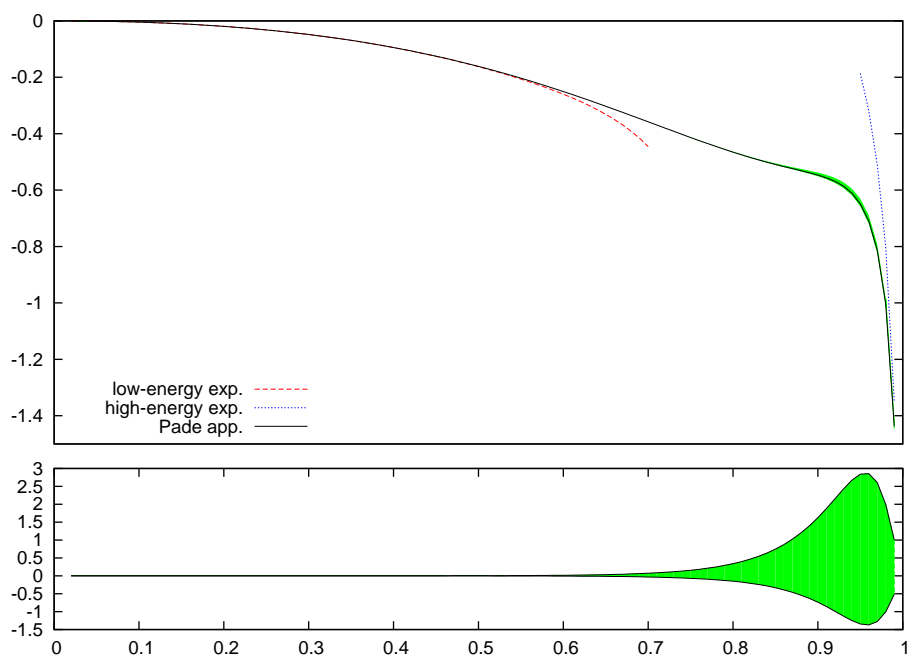

Figure 1: Padé approximation for $\Pi^{(4)}\left(-\frac{x^{2}}{1-x} m_{\mu}^{2}\right)$. We show in the top half the approximants and in the bottom half the relative error with respect to the local mean of all approximants obtained.

becomes more involved. Our strategy will be to incorporate as much of the threshold information as possible into the logarithmic function $\Pi_{\log }^{(3)}(z)$, so that all constraints are imposed at $\omega=0$. The resulting system can be solved very efficiently using well-established techniques of one-point Padé approximation.

As in the four-loop case we first split the polarization function into two parts, using

$$
\begin{aligned}
& \Pi_{\log }^{(3)}(z)=\sum_{i \geq 0, j} k_{i j} \mathcal{K}_{i j}^{(3)}(z)+\sum_{m, n} d_{m n} \mathcal{D}_{m n}^{(3)}(z) \\
& \mathcal{K}_{i j}^{(3)}(z)=\Pi^{(2)}(z)^{i} G(z)^{j} \times\left\{\begin{array}{cl}
(1-z)^{j} & \text { for } j>0 \\
1 & \text { otherwise }
\end{array}\right. \\
& \mathcal{D}_{i j}^{(3)}(z)=(z G(z))^{m}(1-1 / z)^{\left\lceil\frac{m}{2}\right\rceil} \frac{1}{z^{n}}
\end{aligned}
$$

to absorb the logarithms and the threshold singularity. Since we expect to obtain sufficiently many different Padé approximants for a reliable error estimate we refrain from introducing additional parameters at this point.

To map all available information from the low- and high-energy expansions 
onto $\omega=0$, we define

$$
\begin{aligned}
P_{30}(\omega) & =z(\omega)^{31}\left(\Pi_{\mathrm{reg}}^{(3)}(z(\omega))-\sum_{i=0}^{30} \frac{H_{i}^{(3)}}{z(\omega)^{i}}\right), \\
H_{i}^{(3)} & =\left.\frac{1}{i !}\left(\frac{\partial}{\partial(1 / z)}\right)^{i} \Pi_{\mathrm{reg}}^{(3)}(z)\right|_{z \rightarrow-\infty} .
\end{aligned}
$$

The Taylor approximant is fixed by imposing

$$
p_{61,0}^{(i)}(0)=P_{30}^{(i)}(0), \quad i=0, \ldots, 61 .
$$

We can deduce all further approximants with $n+m=61$ and $n+m=60$ using Baker's recursion formula [49]1]

$$
\begin{array}{rlrl}
p_{n-\frac{j}{2}, \frac{j}{2}} & =\frac{\hat{\eta}_{j}}{\hat{\theta}_{j}}=\frac{\hat{\eta}_{j-2}-\omega \hat{\eta}_{j-1}}{\hat{\theta}_{j-2}-\omega \hat{\theta}_{j-1}} & j \text { even }, \\
p_{n-\left\lceil\frac{j}{2}\right\rceil,\left\lfloor\frac{j}{2}\right\rfloor}=\frac{\hat{\eta}_{j}}{\hat{\theta}_{j}}=\frac{\hat{\eta}_{j-2}-\hat{\eta}_{j-1}}{\hat{\theta}_{j-2}-\hat{\theta}_{j-1}} & j \text { odd },
\end{array}
$$

where the numerator $\hat{\eta}_{j}$ of the Padé approximant is normalised as in Eq. (27).

It should be noted that the known threshold constant at order $1-z$ is not included in the approximants. We find however that the information from the threshold region has virtually no effect on the reconstructed polarization functions in the Euclidean regime $z<0$. This is contrary to the four-loop case, where the approximants are far less constrained by the low- and high-energy expansions.

\section{The anomalous magnetic moment of the muon at five loops}

The QED corrections to the anomalous magnetic moment can be calculated in perturbation theory and can thus be written in form of a power series in the fine structure constant $\alpha$

$$
a_{\mu}=\sum_{k=1}^{\infty}\left(\frac{\alpha}{\pi}\right)^{k} a_{\mu}^{(2 k)}
$$

where $a_{\mu}^{(2 k)}$ can be further decomposed - following the conventions in Ref. [8] as

$$
a_{\mu}^{(2 k)}=A_{1}^{(2 k)}+A_{2}^{(2 k)}\left(m_{e} / m_{\mu}\right)+A_{2}^{(2 k)}\left(m_{\tau} / m_{\mu}\right)+A_{3}^{(2 k)}\left(m_{e} / m_{\mu}, m_{\tau} / m_{\mu}\right) .
$$

\footnotetext{
${ }^{1}$ Note that the denominator in the first equation of Eq. (13) in Ref. [49] contains an obvious typo. The proper expression is $\bar{\eta}_{2 j-1} \theta_{2 j-2}(x)-x \bar{\eta}_{2 j-2} \theta_{2 j-1}(x)$.
} 


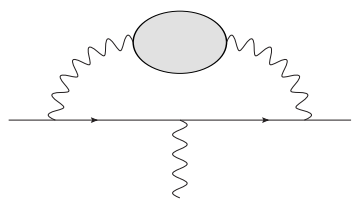

Figure 2: Prototype diagram

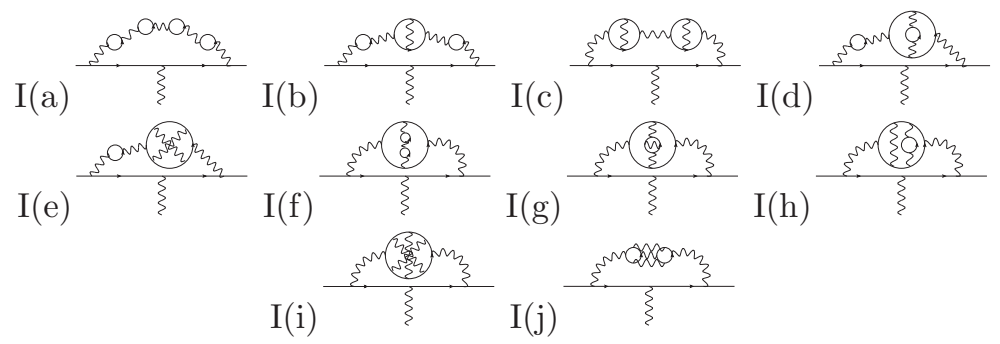

Figure 3: Classes of diagrams accessible by the used method.

$A_{1}^{(2 k)}$ contains the universal contributions, which in case of the muon anomalous magnetic moment only contain muon loops. The diagrams contributing to $A_{2}^{(2 k)}\left(m_{e} / m_{\mu}\right)$ and $A_{2}^{(2 k)}\left(m_{\tau} / m_{\mu}\right)$ have at least one electron or tau loop, respectively. In $A_{3}^{(2 k)}\left(m_{e} / m_{\mu}, m_{\tau} / m_{\mu}\right)$ contributions from diagrams with both electron and tau loops are collected. In this paper we are mainly interested in contributions to $A_{2}^{(2 k)}\left(m_{e} / m_{\mu}\right)$ without any muon loops.

The contributions to the anomalous magnetic moment of the muon due to photon polarization effects can be calculated (cp. Fig. 2) by using

$$
a_{\mu}=\frac{\alpha}{\pi} \int_{0}^{1} \mathrm{~d} x(1-x)\left[-\Pi\left(s_{x}\right)\right], s_{x}=-\frac{x^{2}}{1-x} m_{\mu}^{2} .
$$

Multiple insertions of one-particle irreducible vacuum polarization can be obtained by using

$$
a_{\mu}=\frac{\alpha}{\pi} \int_{0}^{1} \mathrm{~d} x(1-x)\left[-\Pi^{1 \mathrm{PI}}\left(s_{x}\right)\right]^{n},
$$

leading to e.g. at two-loop order

$$
a_{\mu}=\frac{\alpha}{\pi} \int_{0}^{1} \mathrm{~d} x(1-x)\left[-\Pi^{2 \mathrm{~L}}\left(s_{x}\right)+2 \Pi^{1 \mathrm{~L}}\left(s_{x}\right) \Pi^{1 \mathrm{~L}}\left(s_{x}\right)\right] .
$$

The classes of diagrams accessible by this method are shown in Fig. 3

In the following, we compare the results obtained in our analysis with results from previous calculations. The numbers shown are obtained by numerically integrating over the best available approximation. In case there are several equivalent approximations the result is obtained by taking the mean of all values obtained. The errors are then calculated by taking the difference between the mean and the smallest and largest values obtained, respectively. 


\begin{tabular}{|c|c|c|c|c|}
\hline & this work & Ref. [17] & Refs. $[21,50-52]$ & \\
\hline$\overline{\mathrm{I}(\mathrm{a})}$ & 20.142813 & 20.1832 & $20.14293(23)$ & $\overline{[50]}$ \\
\hline $\mathrm{I}(\mathrm{b})$ & 27.690061 & 27.7188 & $27.69038(30)$ & [50] \\
\hline $\mathrm{I}(\mathrm{c})$ & 4.742149 & 4.81759 & $4.74212(14)$ & $\overline{[50]}$ \\
\hline $\mathrm{I}(\mathrm{d})+\mathrm{I}(\mathrm{e})$ & 6.241470 & 6.11777 & $6.24332(101)(70)$ & [50] \\
\hline $\mathrm{I}(\mathrm{e})$ & -1.211249 & -1.33141 & $-1.20841(70)$ & [50] \\
\hline $\mathrm{I}(\mathrm{f})+\mathrm{I}(\mathrm{g})+\mathrm{I}(\mathrm{h})$ & $4.4469_{-4}^{+6}$ & 4.39131 & $4.44668(9)(23)(59)$ & {$[50,51]$} \\
\hline $\mathrm{I}(\mathrm{i})$ & $0.0746_{-19}^{+8}$ & 0.25237 & $0.0871(59)$ & [21] \\
\hline $\mathrm{I}(\mathrm{j})$ & $-1.2469_{-3}^{+4}$ & -1.21429 & $-1.24726(12)$ & {$[52]$} \\
\hline
\end{tabular}

Table 1: Results for $A_{2}^{(10)}\left(m_{e} / m_{\mu}\right)$ with pure electronic insertions. Please note that the authors of Ref. 17] only used the asymptotic form of $\Pi(s)$ and did not provide any error estimate.

\begin{tabular}{c||c|c||} 
& this work & Ref. [8] \\
\hline $\mathrm{I}(\mathrm{a})$ & 22.566976 & $22.566973(3)$ \\
\hline $\mathrm{I}(\mathrm{b})$ & 30.667093 & $30.667091(3)$ \\
\hline $\mathrm{I}(\mathrm{c})$ & 5.141395 & $5.141395(1)$ \\
\hline $\mathrm{I}(\mathrm{e})$ & -0.931839 & $-0.9312(24)$
\end{tabular}

Table 2: Results for $A_{2}^{(10)}\left(m_{e} / m_{\mu}\right)$ including electronic and muonic contributions.

\begin{tabular}{l||l|l||} 
& this work & Ref. [9] \\
\hline \hline $\mathrm{I}(\mathrm{a})$ & 0.000471 & $0.00047094(6)$ \\
\hline $\mathrm{I}(\mathrm{b})$ & 0.007010 & $0.0070108(7)$ \\
\hline $\mathrm{I}(\mathrm{c})$ & 0.023467 & $0.023468(2)$ \\
\hline $\mathrm{I}(\mathrm{d})+\mathrm{I}(\mathrm{e})$ & 0.014093 & $0.014098(5)(4)$ \\
\hline $\mathrm{I}(\mathrm{e})$ & 0.010291 & $0.010296(4)$ \\
\hline $\mathrm{I}(\mathrm{f})+\mathrm{I}(\mathrm{g})+\mathrm{I}(\mathrm{h})$ & 0.037795 & $0.037833(20)(6)(13)$ \\
\hline $\mathrm{I}(\mathrm{i})$ & 0.017167 & $0.01747(11)$ \\
\hline $\mathrm{I}(\mathrm{j})$ & 0.000390 & $0.0003975(18)$
\end{tabular}

Table 3: Results for the universal contributions $A_{1}^{(10)}$. 
For classes $I(a)-I(j)$ we present our results for the case of purely electronic vacuum polarization insertions in Tab. 1. We compare our results with the values obtained in Ref. [17], which relies only on the leading asymptotics of $\Pi\left(q^{2}\right)$, and results obtained by purely numerical calculations.

Since the results for classes I(a)-I(c) are obtained by numerical integrating the exact analytical expression for the one- and two-loop vacuum polarization they are exact.

In the case of classes I(d) and I(e) the used three-loop Padés are highly constrained by a large number of terms in the low- and high-energy expansion. Thus the error from the spread between different approximants is negligible. As can be seen the results are in good agreement with the results obtained in Ref. [50] and the analysis in Ref. [17] can clearly be improved by including sub-leading contributions.

In case of classes I(f)-I(j) the used four-loop Padés are less precise but also here we find good agreement within the quoted errors with the results obtained in Ref. 8]. In all cases one finds a significant improvement when comparing to the leading logarithmic approximation used in Ref. [17].

For classes I(a)-(c) and I(e) we can obtain the full result for $A_{2}^{(10)}\left(m_{e} / m_{\mu}\right)$ including muonic contributions. These results are presented in Tab. 2 In Tab. 3 we present our results for the universal corrections and compare with the results given in Ref. [9]. In both cases the discussion as for the purely electronic contributions can essentially be repeated and also here good agreement with results available in the literature is observed.

As a check of our setup we repeated the analysis of Ref. [15] and found complete agreement with their work.

\section{Conclusions}

We presented results for a certain set of five-loop contributions to the anomalous magnetic moment of the muon that stem from corrections to the vacuum polarization of the photon. We have shown that an improved treatment of the vacuum polarization, including more than its asymptotic form, leads to a significantly better agreement with results obtained by purely numerical methods. It can be clearly seen that for certain classes of diagrams the asymptotic form of the vacuum polarization function is not sufficient and power suppressed terms play an important role and have to be included in the analysis.

\section{Acknowledgements}

We like to thank K.G. Chetyrkin and J.H. Kühn for initiating the project, fruitful discussions, and reading of the manuscript. In addition, we thank K.G. Chetyrkin for valuable advice on computing the vacuum polarization function in high-energy limit. The work of P.B. was supported by RFBR grant 1102-01196. P.M. has been supported in part by DFG Sonderforschungsbereich Transregio 9, Computergestützte Theoretische Teilchenphysik, and by the EU Network LHCPHENOnet PITN-GA-2010-264564. 


\section{References}

[1] J. S. Schwinger, On Quantum electrodynamics and the magnetic moment of the electron, Phys.Rev. 73 (1948) 416-417. doi:10.1103/PhysRev.73.416.

[2] A. Petermann, Fourth order magnetic moment of the electron, Helv.Phys.Acta 30 (1957) 407-408.

[3] C. M. Sommerfield, Magnetic Dipole Moment of the Electron, Phys.Rev. 107 (1957) 328-329. doi:10.1103/PhysRev.107.328.

[4] T. Kinoshita, New value of the alpha**3 electron anomalous magnetic moment, Phys.Rev.Lett. 75 (1995) 4728-4731. doi:10.1103/PhysRevLett.75.4728

[5] S. Laporta, E. Remiddi, The Analytical value of the electron (g2) at order alpha**3 in QED, Phys.Lett. B379 (1996) 283-291. arXiv:hep-ph/9602417, doi:10.1016/0370-2693(96)00439-X.

[6] K. Melnikov, T. v. Ritbergen, The Three loop relation between the MS-bar and the pole quark masses, Phys.Lett. B482 (2000) 99-108. arXiv:hep-ph/9912391, doi:10.1016/S0370-2693(00)00507-4.

[7] P. Marquard, L. Mihaila, J. Piclum, M. Steinhauser, Relation between the pole and the minimally subtracted mass in dimensional regularization and dimensional reduction to three-loop order, Nucl.Phys. B773 (2007) 1-18. arXiv:hep-ph/0702185, doi:10.1016/j.nuclphysb.2007.03.010

[8] T. Aoyama, M. Hayakawa, T. Kinoshita, M. Nio, Complete Tenth-Order QED Contribution to the Muon g-2, Phys.Rev.Lett. 109 (2012) 111808. arXiv:1205.5370, doi:10.1103/PhysRevLett.109.111808

[9] T. Aoyama, M. Hayakawa, T. Kinoshita, M. Nio, Tenth-Order QED Contribution to the Electron g-2 and an Improved Value of the Fine Structure Constant, Phys.Rev.Lett. 109 (2012) 111807. arXiv:1205.5368, doi:10.1103/PhysRevLett.109.111807.

[10] B. Lautrup, E. de Rafael, The anomalous magnetic moment of the muon and short-distance behaviour of quantum electrodynamics, Nucl.Phys. B70 (1974) 317-350. doi:10.1016/0550-3213(74)90481-7.

[11] T. Kinoshita, H. Kawai, Y. Okamoto, Asymptotic photon propagator in massive QED and the muon anomalous magnetic moment, Phys.Lett. B254 (1991) 235-240. doi:10.1016/0370-2693(91)90427-R.

[12] H. Kawai, T. Kinoshita, Y. Okamoto, Asymptotic photon propagator and higher order QED Callan-Symanzik beta function, Phys.Lett. B260 (1991) 193-198. doi:10.1016/0370-2693(91)90990-8. 
[13] R. Faustov, A. Kataev, S. Larin, V. Starshenko, THE ANALYTICAL CONTRIBUTION OF THE THREE LOOP DIAGRAMS WITH TWO FERMION CIRCLES TO THE PHOTON PROPAGATOR AND THE MUON ANOMALOUS MAGNETIC MOMENT, Phys.Lett. B254 (1991) 241-246. doi:10.1016/0370-2693(91)90428-S.

[14] D. J. Broadhurst, A. Kataev, O. Tarasov, Analytical on-shell QED results: Three loop vacuum polarization, four loop Beta function and the muon anomaly, Phys.Lett. B298 (1993) 445-452. arXiv:hep-ph/9210255, doi:10.1016/0370-2693(93)91849-I.

[15] P. Baikov, D. J. Broadhurst, Three loop QED vacuum polarization and the four loop muon anomalous magnetic momentarXiv:hep-ph/9504398.

[16] S. Laporta, The Analytical contribution of some eighth order graphs containing vacuum polarization insertions to the muon (g-2) in QED, Phys.Lett. B312 (1993) 495-500. arXiv:hep-ph/9306324, doi:10.1016/0370-2693(93)90988-T

[17] P. Baikov, K. Chetyrkin, J. Kühn, C. Sturm, K. Chetyrkin, et al., The relation between the QED charge renormalized in MSbar and on-shell schemes at four loops, the QED on-shell beta-function at five loops and asymptotic contributions to the muon anomaly at five and six loops, Nucl.Phys. B867 (2013) 182-202. arXiv:1207.2199, doi:10.1016/j.nuclphysb.2012.09.018.

[18] R. Lee, P. Marquard, A. V. Smirnov, V. A. Smirnov, M. Steinhauser, Four-loop corrections with two closed fermion loops to fermion self energies and the lepton anomalous magnetic moment, JHEP 1303 (2013) 162. arXiv:1301.6481, doi:10.1007/JHEP03(2013) 162

[19] F. Jegerlehner, A. Nyffeler, The Muon g-2, Phys.Rept. 477 (2009) 1-110. arXiv:0902.3360, doi:10.1016/j.physrep.2009.04.003

[20] R. Barbieri, E. Remiddi, Electron and Muon 1/2(g-2) from Vacuum Polarization Insertions, Nucl.Phys. B90 (1975) 233. doi:10.1016/0550-3213(75)90645-8.

[21] T. Aoyama, M. Hayakawa, T. Kinoshita, M. Nio, Proper EighthOrder Vacuum-Polarization Function and its Contribution to the TenthOrder Lepton g-2, Phys.Rev. D83 (2011) 053003. arXiv:1012.5569, doi:10.1103/PhysRevD.83.053003

[22] A. Maier, P. Maierhöfer, P. Marquard, A. Smirnov, Low energy moments of heavy quark current correlators at four loops, Nucl.Phys. B824 (2010) 1-18. arXiv:0907.2117, doi:10.1016/j.nuclphysb.2009.08.011.

[23] P. Nogueira, Automatic feynman graph generation, J. Comput. Phys. 105 (1993) 279-289. 
[24] T. Seidensticker, Automatic application of successive asymptotic expansions of Feynman diagramsarXiv:hep-ph/9905298,

[25] R. Harlander, T. Seidensticker, M. Steinhauser, Complete corrections of Order alpha alpha-s to the decay of the $\mathrm{Z}$ boson into bottom quarks, Phys.Lett. B426 (1998) 125-132. arXiv:hep-ph/9712228, doi:10.1016/S0370-2693(98)00220-2

[26] J.A.M.Vermaseren, New features of FORM URL math-ph/0010025

[27] P. Marquard, D. Seidel, Crusher, unpublished.

[28] S. Laporta, High precision calculation of multiloop Feynman integrals by difference equations, Int.J.Mod.Phys. A15 (2000) 5087-5159. arXiv:hep-ph/0102033, doi:10.1016/S0217-751X(00)00215-7.

[29] K. Chetyrkin, M. Faisst, C. Sturm, M. Tentyukov, epsilon-finite basis of master integrals for the integration-by-parts method, Nucl.Phys. B742 (2006) 208-229. arXiv:hep-ph/0601165, doi:10.1016/j.nuclphysb.2006.02.030.

[30] Y. Schroder, M. Steinhauser, Four-loop decoupling relations for the strong coupling, JHEP 0601 (2006) 051. arXiv:hep-ph/0512058, doi:10.1088/1126-6708/2006/01/051.

[31] S. Laporta, High precision epsilon expansions of massive four loop vacuum bubbles, Phys.Lett. B549 (2002) 115-122. arXiv:hep-ph/0210336 doi:10.1016/S0370-2693(02) 02910-6.

[32] K. Chetyrkin, J. H. Kuhn, P. Mastrolia, C. Sturm, Heavy-quark vacuum polarization: First two moments of the $\mathrm{O}\left(\right.$ alpha**3(s) $\left.\mathrm{n}^{* *} 2(\mathrm{f})\right)$ contribution, Eur.Phys.J. C40 (2005) 361-366. arXiv:hep-ph/0412055, doi:10.1140/epjc/s2005-02151-y

[33] B. A. Kniehl, A. V. Kotikov, Calculating four-loop tadpoles with one non-zero mass, Phys.Lett. B638 (2006) 531-537. arXiv:hep-ph/0508238, doi:10.1016/j.physletb.2006.04.057.

[34] Y. Schroder, M. Steinhauser, Four-loop singlet contribution to the rho parameter, Phys.Lett. B622 (2005) 124-130. arXiv:hep-ph/0504055, doi:10.1016/j.physletb.2005.06.085.

[35] E. Bejdakic, Y. Schroder, Hypergeometric representation of a fourloop vacuum bubble, Nucl.Phys.Proc.Suppl. 160 (2006) 155-159. arXiv:hep-ph/0607006, doi:10.1016/j.nuclphysbps.2006.09.040

[36] B. A. Kniehl, A. V. Kotikov, Heavy-quark QCD vacuum polarisation function: Analytical results at four loops, Phys.Lett. B642 (2006) 68-71. arXiv:hep-ph/0607201, doi:10.1016/j.physletb.2006.09.008. 
[37] B. Kniehl, A. Kotikov, A. Onishchenko, O. Veretin, Strongcoupling constant with flavor thresholds at five loops in the antiMS scheme, Phys.Rev.Lett. 97 (2006) 042001. arXiv:hep-ph/0607202, doi:10.1103/PhysRevLett.97.042001.

[38] P. Baikov, A Practical criterion of irreducibility of multi-loop Feynman integrals, Phys.Lett. B634 (2006) 325-329. arXiv:hep-ph/0507053, doi:10.1016/j.physletb.2006.01.052.

[39] P. Baikov, Explicit solutions of the three loop vacuum integral recurrence relations, Phys.Lett. B385 (1996) 404-410. arXiv: hep-ph/9603267, doi:10.1016/0370-2693(96)00835-0.

[40] P. Baikov, K. Chetyrkin, Four Loop Massless Propagators: An Algebraic Evaluation of All Master Integrals, Nucl.Phys. B837 (2010) 186-220. arXiv:1004.1153, doi:10.1016/j.nuclphysb.2010.05.004

[41] A. Hoang, M. Beneke, K. Melnikov, T. Nagano, A. Ota, et al., Top - antitop pair production close to threshold: Synopsis of recent NNLO results, Eur.Phys.J.direct C2 (2000) 1. arXiv:hep-ph/0001286.

[42] A. H. Hoang, V. Mateu, S. Mohammad Zebarjad, Heavy Quark Vacuum Polarization Function at $O\left(\alpha_{s}^{2}\right)$ and $O\left(\alpha_{s}^{3}\right)$, Nucl. Phys. B813 (2009) 349369. arXiv:0807.4173, doi:10.1016/j.nuclphysb.2008.12.005.

[43] Y. Kiyo, A. Maier, P. Maierhöfer, P. Marquard, Reconstruction of heavy quark current correlators at $\mathcal{O}\left(\alpha_{s}^{3}\right)$, Nucl.Phys. B823 (2009) 269-287. arXiv:0907.2120, doi:10.1016/j.nuclphysb.2009.08.010

[44] G. A. Baker, Essentials of Padé Approximants, Academic Press, Inc., 1975.

[45] D. J. Broadhurst, J. Fleischer, O. V. Tarasov, Two loop two point functions with masses: Asymptotic expansions and Taylor series, in any dimension, Z. Phys. C60 (1993) 287-302. arXiv:hep-ph/9304303, doi:10.1007/BF01474625.

[46] J. Fleischer, O. V. Tarasov, Calculation of Feynman diagrams from their small momentum expansion, Z. Phys. C64 (1994) 413-426. arXiv:hep-ph/9403230, doi:10.1007/BF01560102.

[47] D. J. Broadhurst, et al., Two loop gluon condensate contributions to heavy quark current correlators: Exact results and approximations, Phys. Lett. B329 (1994) 103-110. arXiv:hep-ph/9403274, doi:10.1016/0370-2693(94)90524-X.

[48] K. G. Chetyrkin, J. H. Kühn, M. Steinhauser, Heavy Quark Vacuum Polarisation to Three Loops, Phys. Lett. B371 (1996) 93-98. arXiv:hep-ph/9511430, doi:10.1016/0370-2693(95) 01593-0. 
[49] G. A. Baker Jr., J. L. Gammel, The Padé Approximant in Theoretical Physics, Vol. 71 of Mathematics in Science and Engineering, Academic Press, Inc., 1970, Ch. 1, pp. 1-38.

[50] T. Kinoshita, M. Nio, The Tenth-order QED contribution to the lepton g-2: Evaluation of dominant alpha**5 terms of muon g-2, Phys.Rev. D73 (2006) 053007. arXiv:hep-ph/0512330, doi:10.1103/PhysRevD.73.053007.

[51] T. Aoyama, M. Hayakawa, T. Kinoshita, M. Nio, Tenth-Order Lepton Anomalous Magnetic Moment: Second-Order Vertex Containing Two Vacuum Polarization Subdiagrams, One Within the Other, Phys.Rev. D78 (2008) 113006. arXiv:0810.5208, doi:10.1103/PhysRevD.78.113006.

[52] T. Aoyama, M. Hayakawa, T. Kinoshita, M. Nio, N. Watanabe, Eighth-Order Vacuum-Polarization Function Formed by Two Light-byLight-Scattering Diagrams and its Contribution to the Tenth-Order Electron g-2, Phys.Rev. D78 (2008) 053005. arXiv:0806.3390, doi:10.1103/PhysRevD.78.053005 